\title{
Diffusion Weighted MRI - The Diagnostic Modality to Differentiate Renal Pseudotumor from Renal Cell Carcinoma in Chronic Kidney Disease: A Case Report
}

\author{
Anmol Modi ${ }^{1}$, Chanabasappa Chavadi', Pallavi Patri ${ }^{2}$ \\ ${ }^{1}$ Senior Resident, Department of Radiology, ${ }^{2}$ Consultant, Department of Nephrologist, Columbia Asia Hospital, Survey No. 45/2 ward Police Station, No 150, \\ Sarjapur Main Rd, Ambalipura, Bellandur, Bengaluru, Karnataka 560102.
}

\section{Abstract}

Renal pseudo tumors are rare occurence in patients with chronic kidney disease, most of the times it is detected incidentally on imaging and mimics a renal cell carcinoma. It is imperative to exclude renal cell carcinoma in these patients. A 38-year-old female P2L2A0 presented with menorrhagia, Blood laboratory test revealed elevated creatinine level $2.2 \mathrm{mg} / \mathrm{dl}$. Other blood parameters showed raised urea levels -52 , urine protein to creatinine-1.3 and urine protein 1+. Follow up lab values for a period of 3 months revealed persistently raised urea and creatinine levels. MRI with contrast demonstrates small sized bilateral kidneys with parenchymal thinning and multiple well defined lobulated partially exophytic mass lesions, homogenously mildly hyperintense on T2W images, and isointense on T1W images, lesion demonstrated no diffusion restriction on DWI images.

Keywords: Chronic kidney disease, renal cell carcinoma, renal pseudotumor, diffusion weighted MRI.

Corresponding Author: Dr. Anmol Modi, Senior Resident, Department of Radiology, Columbia Asia Hospital, Survey No. 45/2 ward Police Station, No 150, Sarjapur Main Rd, Ambalipura, Bellandur, Bengaluru, Karnataka 560102.

Received: June 2019

Accepted: June 2019

\section{Introduction}

Chronic kidney disease (CKD) is carrying the major burden of morbidity and mortality globally, Hypertension, diabetes mellitus and cardiovascular diseases are major primary risk factors to CKD. Renal pseudo tumors are rare occurence in patients with chronic kidney disease, most of the times it is detected incidentally on imaging and mimics a renal cell carcinoma. It is imperative to exclude renal cell carcinoma in these patients. We present case of renal pseudotumor in a female with NSAID's induced chronic kidney disease.

\section{Case Report}

A 38-year-old female P2L2A0 presented with menorrhagia, Blood laboratory test revealed elevated creatinine level $2.2 \mathrm{mg} / \mathrm{dl}$. Other blood parameters showed raised urea levels -52 , urine protein to creatinine- 1.3 and urine protein $1+$. Follow up lab values for a period of 3 months revealed persistently raised urea and creatinine levels. Patient gives a history of using combiflam regularly past a year for chronic persistent headache, clinically patient was suspected for NSAIDs induced chronic kidney disease. Patient family history did not reveal any significant point of note.

Patient was referred to our department for radiological investigations.

Ultrasonography showed small sized kidneys with bilateral increased renal parenchymal echogenicity with loss of corticomedullary differentiation and an isoechoic lesion in the left kidney with internal vascularity.

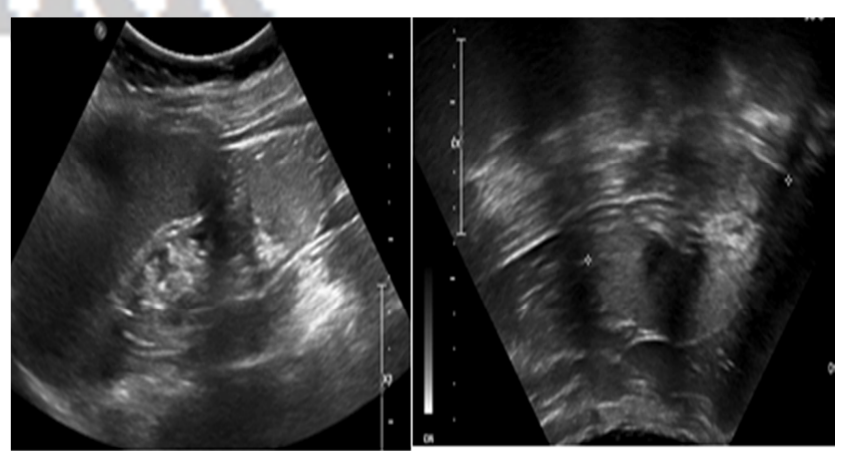

Figure 1: USG revealed small sized left kidney with parenchymal thinning (white arrow) and a well-defined isoechoic lesion (blue arrow).

On non-contrast CT, the kidneys were small sized bilaterally with parenchymal thinning and multiple partially exophytic well defined lobulated isodense mass lesions. No evidence of calcification on cystic areas. Lesions were compressing the calyceal system. No hydronephrosis was seen. No invasion into the renal veins. Rest of the organs were unremarkable.

MRI with contrast demonstrates small sized bilateral kidneys with parenchymal thinning and multiple well 
defined lobulated partially exophytic mass lesions, homogenously mildly hyperintense on T2W images, and isointense on T1W images, lesion demonstrated no diffusion restriction on DWI images. No areas of susceptibility on GRE. Lesions are demonstrating homogenous post contrast enhancement similar to enhancement of intervening renal parenchyma. No evidence of hydronephrosis.

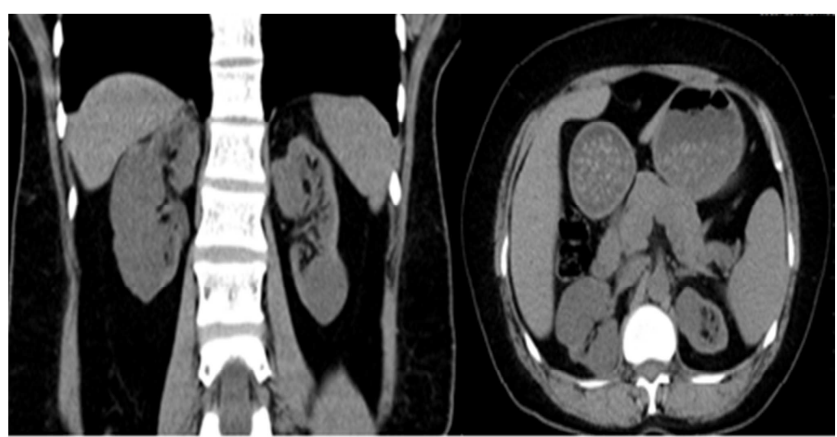

Figure 2: Non-contrast CT coronal image demonstrate small kidneys with parenchymal thinning (thick arrow) and multiple partially exophytic hypodense to isodense soft tissue attenuation mass lesions (thin arrows).
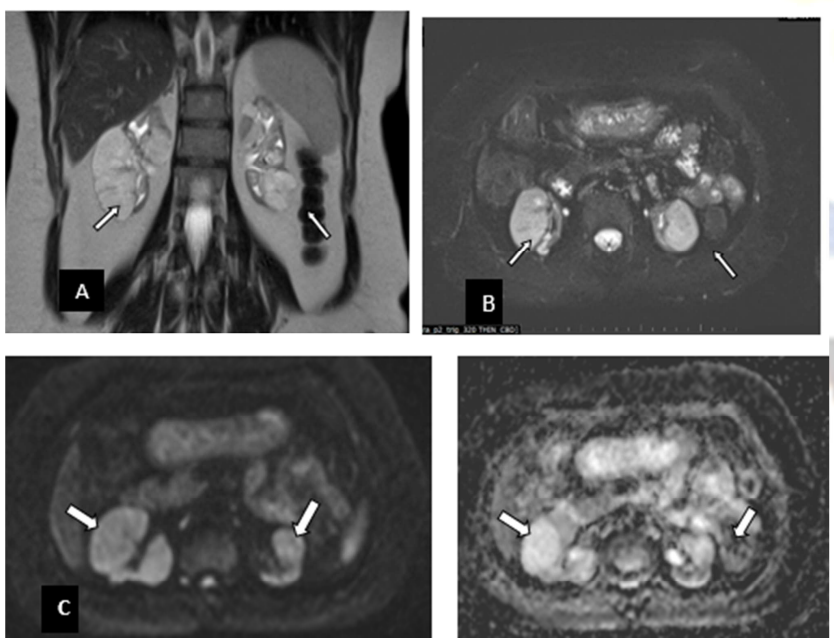

Figure 3: Contrast enhanced MRI, (A) Coronal T2 HASTE, (B) Axial T2 HASTE FAT SAT, (C) Diffusion weighted imaging (DWI) and (D) Apparent diffusion coefficient (ADC) demonstrating changes of chronic kidney disease, multiple well defined hyperintense mass lesions (thin arrows) involving both kidneys. Lesions are hyperintense on DWI and ADC (thick arrows), suggesting no diffusion restriction. Homogenous contrast enhancement is seen on post contrast image.

\section{Discussion}

Renal pseudotumors mimics renal neoplasms on imaging. Congenital causes include hypertrophied column of Bertin, fetal lobulation, dromedary hump and cross-fused ectopic kidney. In patients with chronic renal disease, renal pseudotumor occurs as a result of nodular compensatory hypertrophy where regenerating nodules appear as renal masses. Other acquired causes are renal sinus lipomatosis, xanthogranulomatous pyelonephritis, infections such as renal tuberculosis and actinomycosis, Wegener granulomatosis, and arteriovenous malformation.

Multiple modalities like ultrasonography, CT, radionuclide scan and MRI can be used for differentiating renal pseudotumors from renal neoplasms. However, in patients with chronic renal disease, IV contrast cannot be administered due to deranged renal parameters. Ultrasonography and non-contrast CT cannot reliably differentiate renal pseudotumors from renal neoplasms. Renal pseudotumors demonstrate no diffusion restriction on DWI while renal cell carcinomas have diffusion restriction. Therefore non-contrast MRI with diffusion weighted imaging serves as a problem solving tool to differentiate between these two conditions.

In our case, we have performed ultrasonography and noncontrast CT which were inconclusive. On non-contrast MRI with diffusion weighted imaging, we could reliably conclude as renal pseudotumor due to absence of diffusion restriction. The patient was then followed up with ultrasound at regular interval for upto 6 months and the lesions were stable in size and morphology.

\section{Conclusion}

Use of non-contrast MRI with diffusion weighted imaging can reliably differentiate renal pseudotumors from renal cell carcinoma in patients with chronic renal discase and precluding the need for further imaging and invasive procedures like renal biopsy to arrive at a diagnosis.

References

1. Indiran $\mathrm{V}$. Magnetic resonance imaging as problem-solving tool in renal pseudotumor in chronic kidney disease: A case of nodular compensatory hypertrophy. Indian J Nephrol 2016;26:470-1

2. Pseudotumours in chronic kidney disease: Can diffusion-weighted MRI rule out malignancy: European Journal of Radiology; ISSN 0720048X; : CODEN EJRADR; v. 82(11); p. 1870-1876

Copyright: (C) the author(s), publisher. Asian Journal of Medical Radiological Research is an Official Publication of "Society for Health Care \& Research Development”. It is an open-access article distributed under the terms of the Creative Commons Attribution Non-Commercial License, which permits unrestricted non-commercial use, distribution, and reproduction in any medium, provided the original work is properly cited.

How to cite this article: Modi A, Chavadi C, Patri P. Diffusion Weighted MRI - The Diagnostic Modality to Differentiate Renal Pseudotumor from Renal Cell Carcinoma in Chronic Kidney Disease: A Case Report. Asian J. Med. Radiol. Res. 2019;7(1):97-98. DOI: dx.doi.org/10.21276/ajmrr.2019.7.1.21

Source of Support: Nil, Conflict of Interest: None declared. 